\title{
Treatment of refractory auditory hallucinations in schizophrenia by rTMS: Positive impact of negative double blind controlled study
}

\author{
Noomane Bouaziz , Djedia Sidhoumi, Sonia Braha, Ghada Kallel, \\ Palmyre Schenin-King Andrianisaina, Virginie Moulier, René Benadhira, Dominique Januel \\ Clinical Research Unit, Neuilly Sur Marne, France \\ Email: "bouaziznoomane@gmail.com
}

Received 21 August 2013; revised 20 September 2013; accepted 28 September 2013

Copyright (c) 2014 Noomane Bouaziz et al. This is an open access article distributed under the Creative Commons Attribution License, which permits unrestricted use, distribution, and reproduction in any medium, provided the original work is properly cited. In accordance of the Creative Commons Attribution License all Copyrights (C) 2014 are reserved for SCIRP and the owner of the intellectual property Noomane Bouaziz et al. All Copyright (C) 2014 are guarded by law and by SCIRP as a guardian.

\section{ABSTRACT}

Background: Several studies reported on the effectiveness of $1 \mathrm{~Hz}$ repeated transcranial magnetic stimulation (rTMS) over the temporoparietal cortex (TPC) on refractory auditory hallucinations (AH) in schizophrenia but little is known about the long-term therapeutic effect of this tool. Aim: The aim of this study was to evaluate rTMS' impact on auditory hallucinations (AH) and cognitive functions in patients with schizophrenia with a three-month follow-up. Methods: In a randomized double blind sham controlled study, 26 patients with refractory schizophrenia received $1 \mathrm{~Hz}$ rTMS applied on TPC for four weeks. rTMS was given to patients over one month with five rTMS sessions each week. Patients were evaluated via AHRS (Auditory Hallucination Rating Scale, Hoffman et al.), PANSS (Positive and Negative Syndrome Scale, Kay et al. 1988), and CGI (Clinical Global Impression) before treatment by rTMS every week during the first month, and every month until the third month. Results: Neither clinical nor cognitive differences were found between rTMS and placebo. Conclusion: $1 \mathrm{~Hz}$ rTMS using parameters mentioned above has no statistically significant effect on AH; although evidences about the potential benefit of rTMS in the treatment of AH negative results suggest the need for further studies in this area.

\section{KEYWORDS}

Schizophrenia; rTMS; Auditory Hallucinations; Temporo-Parietal Cortex

"Corresponding author.

\section{INTRODUCTION}

Repeated transcranial magnetic stimulation is a non-invasive brain stimulation tool, which has shown efficiency in the treatment of some resistant neuropsychiatric disorders, particularly depression and auditory hallucinations in schizophrenia. Five meta-analyses have reported the effectiveness of $1 \mathrm{~Hz}$ repeated transcranial magnetic stimulation (rTMS) over the temporoparietal cortex on refractory auditory hallucinations (AH) in schizophrenia [1-4].

However, little is known about the long-term therapeutic effect of rTMS on auditory hallucinations. Hoffman et al. [5] reported that the average duration of a sustained reduction in AH severity of at least $20 \%$ relative to baseline scores was 19.7 weeks in a study of 50 patients. In a randomized, double blind, controlled study, Poulet et al. [6] reported that after 5 days of low frequency rTMS, 5 of the 7 patients who responded to active treatment maintained clinical improvement for at least two months. Recently, Montagne-Larmurier et al. [7] reported short successful treatment (2 days) of AH in an open pilot study of 11 patients using high frequency rTMS $(20 \mathrm{~Hz})$, which was then maintained over a 6-month follow-up. Thus, the duration of the effect of rTMS would appear to be between 2 and 6 months. However, some recent negative studies with larger samples reduced the global result of this therapeutic tool [8].

In our study, the aim was to evaluate the effectiveness of one month of $1 \mathrm{~Hz}$ neuronavigated rTMS on AH in patients with schizophrenia with a three-month follow-up.

\section{MATERIALS AND METHODS}

\subsection{Subjects}

Twenty-six outpatients, between the ages of 18 and 65, 
meeting the diagnostic criteria for schizophrenia (DSMIV criteria) with refractory auditory hallucination (a failure of treatment with two different antipsychotics, of which at least one was atypical) were included in this study.

The exclusion criteria were: suicide risk, pregnancy, history of seizures, neurosurgery or head trauma, use of pacemaker or intracranial metallic clip, neurological disease, other psychiatric disorder or an unstable medical condition, an estimated IQ of $\leq 80$, current substance abuse, or an inability to provide informed consent. The protocol was approved by the local ethics committee (Ambroise Paré Hospital, France) and all patients provided written informed consent to be included in the study.

\section{2. rTMS Protocol}

This is a randomized double-blind sham-controlled study. Subjects were assigned randomly to undergo TMS treatment $(n=13)$ or sham treatment $(n=13)$. Patients were treated with rTMS, applied to the left temporoparietal cortex (LTPC). The TMS coil was positioned on the left LTPC through a neuronavigation method using the Brainsight Software system (version 1.7.6; Rogue Research Inc., Montréal, Canada). 360 pulses were administered daily at a frequency of $1 \mathrm{~Hz}$, at $100 \%$ of the motor threshold (MT) through 6 trains of 60 seconds, with an inter-train interval of 30 seconds. rTMS was given to patients over the period of one month with five rTMS sessions each week. Each session lasted 8.5 minutes. rTMS stimulation was carried out with a Magstim Super Rapid (INOMED) stimulator system with figure-eight 70$\mathrm{mm}$ coils. The sham coil has the same shape and produces a sound similar to the active one.

The MT was defined as the minimal TMS intensity required to elicit motor evoked potentials (MEPs) of at least $50 \mu \mathrm{V}$ peak-to-peak amplitude, under muscle relaxation, in 5 out of 10 consecutive trials using a MagPro device to deliver TMS and Myto device for EMG recording.

\subsection{Clinical Measures}

All patients were evaluated by AHRS (Auditory Hallucination Rating Scale, Hoffman et al., 2005), PANSS (Positive And Negative Syndrome Scale, Kay et al., 1988), and CGI (Clinical Global Impression) before treatment by rTMS every week during the first month, and every month until the third month.

\subsection{Cognitive Evaluation}

The impact of the TMS on the cognitive functions of all the subjects at day 0 (base line) and after four weeks (M1) was evaluated by a neuropsychological battery containing
7 cognitive tests (California Verbal Learning Test (CVLT), Verbal and visual Digit spans tests (WAIS-R), Trail Making Test A and B, Stroop Test, Wisconsin Card Sorting Test, Hanoi Tower, and Verbal Fluences).

\subsection{Statistical Analysis}

A students-test and chi-squared tests were used to compare the demographic and clinical characteristics of the two groups. To compare the overall effect of treatment over time in the two groups, a set of repeated analysis of variance (one for each dependent variable) using the ANOVA approach was employed with treatment as between group factor and time as the within-subject factor.

\section{RESULTS}

There are no significant differences between active and placebo groups regarding socio-demographic data (age: mean 35.90, (SD 13.25) vs 35.60, (SD 12.35), p > 0.05; sex-ratio (women/men) (\%): 33.35 in active group vs 36.35 in placebo group), illness duration, and psychometric evaluations (AHRS, PANSS, CGI) ( $p>0.05)$.

rTMS treatment was well tolerated and no serious adverse effects were reported by subjects except transient headaches (in three patients with). In this study no difference was found between rTMS treatment and the sham treatment (placebo) in all endpoints in the psychometrics tests (AHRS, PANSS and CGI).

Cognitive assessment will also not show significant differences between active and placebo groups from baseline and a month after the first treatment (M1) and it, for each of the cognitive dimension ( $p>0.05$ ).

\section{DISCUSSION}

Our double blind study does not show any efficacy of rTMS treatment compared with a sham treatment in the occurrence of $\mathrm{AH}$, but it contributes data on the absence of negative effects of this tool on the cognitive functions in the schizophrenic population [9]. This result does enable any conclusion to be drawn concerning efficacy or not of rTMS on AH, due to the relatively small sample size leading to a lack of power (Beta risk). Nevertheless, there are several hypotheses that we will now discuss relating to factors that could have contributed to this negative result.

The first factor contributing to the lack of efficacy could be the low number of stimuli delivered to the patients. In this study 360 stimuli a day were delivered, with a total number of 7200 over four weeks. Most studies describing a positive effect of rTMS on AH used a higher total number of stimuli, ranging from 7920 to 24,000 . The pattern of stimulation used in our study was chosen to deliver the least painful, the safest, and the most comfortable stimulation. We think that there might be a mini- 
mal total number of stimuli (delivered in a short period) required to trigger a benefit of rTMSon $\mathrm{AH}$.

Secondly, the patient's description of his or her own symptoms i.e. AH, could be biased, since many psychotic patients don't consider $\mathrm{AH}$ as a symptom or problem, especially when the symptom has a pleasant and agreeable valence [10]. We suggest that it would be helpful to evaluate the patient's insight before treatment and train them to identify, evaluate, and report his or her own symptoms using psycho-educational sessions to improve the trustworthiness of their reports. Then, the measurable instrument would be optimized to become highly sensitive to any changes in $\mathrm{AH}$.

A further complication is that different studies use different scales (PANSS, AHRS or HCS) to assess the effect of rTMS on AH. Regarding literature HCS seems to be the more scale oriented with positive studies than others: 5 studies, 4 positives [5,11-13] and 1 negative: [14] while AHRS was associated with only 2 positive studies $[2,15]$ and PANSS with one positive study [16].

Thirdly, the stimulation design used in this study consisted of $1 \mathrm{~Hz}$ rTMS with "intertrain breaks" between the train stimulations. We speculate that this "intermittent stimulation" may have had a less beneficial effect than a "continuous stimulation design". Contrary to our conclusions, De Jesus et al. [17] speculated that their negative results were due to their continuous train (without breaks), arguing that most positive publications used stimulation trains with breaks. The influence of breaks in rTMS trains on treatment outcome is not well known. It may be that the physiological effect of an intermittent rTMS train is different from a continuous train. Besides, the theta burst stimulation presented by Huang et al. [18] has shown that its use with continuous design causes an inhibitory effect, while an intermittent theta burst is excitatory. Coupling continuous and intermittent rTMS with neuroexcitability and EEG measures could perhaps show the possible differences in neuromodulatiory effects between these two approaches to stimulation treatment.

Finally, we think that use of rTMS in AH should be integrated in an algorithm. This should not only specify the place rTMS should have in the treatment of $\mathrm{AH}$, but also the adjustment of the rTMS target since several fMRI studies showed great inter-individual differences in activation patterns during hallucination phenomena. In fact, approximately $50 \%$ of the patients' activation during $\mathrm{AH}$ was predominantly present in right-hemispheric areas [19]. That is why we believe that the use of the LTPC as the specific target for rTMS to treat AH should be reconsidered.

Furthermore, the area of the brain targeted by rTMS also needs to be clearly defined since the LTP region appears to have a significant level of inter-individual variability and lack of precision. The use of neuro-navi- gation systems, even if it allows more reproducibility and precision, must more clearly define the area targeted. Indeed, teams that have used this system have targeted a broad range of brain regions, especially when nonfunctional MRI was used.

\section{CONCLUSIONS}

To summarize, the absence of superiority of rTMS versus sham treatment in this study significantly influenced our use of rTMS in the treatment of AH. We suggest that, to optimize its efficacy, rTMS should be delivered powerfully over a short period of time; patients need more support to better recognise their own symptoms to improve the assessment, and that the neuronal target of rTMS needs to be better studied and defined.

As suggested by Slotema et al. [8] following the negative results of rTMS use in their study, we think that it is important that better clarity is included in future studies of rTMS use in the treatment of AH. Furthermore, it would be beneficial to create a decisional algorithm to be used for non-responders to the rTMS classical protocol, for example reconsidering other stimulation paradigms (theta burst stimulation, high-frequency rTMS), other cortical targets (controlateral TP3 for example) or other stimulations techniques (e.g. tDCS).

\section{REFERENCES}

[1] Aleman, A., Sommer, I.E. and Kahn, R.S. (2007) Efficacy of slow repetitive trans-cranial magnetic stimulation in the treatment of resistant auditory hallucinations in schizophrenia: A meta-analysis. Journal of Clinical Psychiatry, 68, 416-421. http://dx.doi.org/10.4088/JCP.v68n0310

[2] Tranulis, C., Sepehry, A.A., Galinowski, A. and Stip, E. (2008) Should we treat auditory hallucinations with repetitive transcranial magnetic stimulation? A metaanalysis. Canadian Journal of Psychiatry, 53, 577-586.

[3] Freitas, C., Fregni, F. and Pascual-Leone, A. (2009) Meta-analysis of the effects of repetitive transcranial magnetic stimulation (rTMS) on negative and positive symptoms in schizophrenia. Schizophrenia Research, 108, 1124. http://dx.doi.org/10.1016/j.schres.2008.11.027

[4] Slotema, C.W., Blom, J.D., Hoek, H.W. and Sommer, I.E.C. (2010) Should we expand the toolbox of psychiatric treatment methods to include repetitive transcranial magnetic stimulation? A meta-analysis of the efficacy of rTMS for psychiatric disorders. Journal of Clinical Psychiatry, 71, 873-884. http://dx.doi.org/10.4088/JCP.08m04872gre

[5] Hoffman, R.E., Boutros, N.N., Berman, R.M., Roessler, E., Belger, A., Krystal, J.H. and Charney, D.S. (1999) Transcranial magnetic stimulation of left temporoparietal cortex in three patients reporting hallucinated "voices". Biological Psychiatry, 46, 130-132. http://dx.doi.org/10.1016/S0006-3223(98)00358-8

[6] Poulet, E., Brunelin, J., Bediou, B., Bation, R., Forgeard, 
L., Dalery, J., d’Amato, T. and Saoud, M. (2005) Slow transcranial magnetic stimulation can rapidly reduce resistant auditory hallucinations in schizophrenia. Biological Psychiatry, 57, 188-191.

http://dx.doi.org/10.1016/j.biopsych.2004.10.007

[7] Montagne-Larmurier, A., Etard, O., Razafimandimby, A., Morello, R. and Dollfus, S. (2009) Two-day treatment of auditory hallucinations by high frequency rTMS guided by cerebral imaging: A 6 month follow-up pilot study. Schizophrenia Research, 113, 77-83. http://dx.doi.org/10.1016/j.schres.2009.05.006

[8] Slotema, C.W., Blom, J.D., de Weijer, A.D., Diederen, K.M., Goekoop, R., Looijestijn, J., Daalman, K., Rijkaart, A.M., Kahn, R.S., Hoek, H.W. and Sommer, I.E. (2011) Can low-frequency repetitive transcranial magnetic stimulation really relieve medication-resistant auditory verbal hallucinations? Negative results from a large randomized controlled trial. Biological Psychiatry, 69, 450456. http://dx.doi.org/10.1016/j.biopsych.2010.09.051

[9] Mittrach, M., Thünker, J., Winterer, G., Agelink, M.W., Regenbrecht, G., Arends, M., Mobascher, A., Kim, S.J., Wölwer, W., Brinkmeyer, J., Gaebel, W. and Cordes, J. (2010) The tolerability of rTMS treatment in schizophrenia with respect to cognitive function. Pharmacopsychiatry, 43, 110-117. http://dx.doi.org/10.1055/s-0029-1242824

[10] Ho, B.C., Flaum, M., Hubbard, W., Arndt, S. and Andreasen, N.C. (2004) Validity of symptom assessment in psychotic disorders: Information variance across different sources of history. Schizophrenia Research, 68, 299-307. http://dx.doi.org/10.1016/j.schres.2003.07.006

[11] Hoffman, R.E., Boutros, N.N., Hu, S., Berman, R.M., Krystal, J.H. and Charney, D.S. (2000) Transcranial magnetic stimulation and auditory hallucinations in schizophrenia. Lancet, 9209, 1073-1075. http://dx.doi.org/10.1016/S0140-6736(00)02043-2

[12] Hoffman, R.E., Gueorguieva, R., Hawkins, K.A., Varanko, M., Boutros, N.N., Wu, Y.T., Carroll, K. and Krystal, J.H. (2005) Temporoparietaltranscranial magnetic stimulation for auditory hallucinations: Safety, efficacy and moderators in a fifty patient sample. Biological Psychiatry, 58, 97-104.

http://dx.doi.org/10.1016/j.biopsych.2005.03.041
[13] Hoffman, R.E., Hampson, M., Wu, K., Anderson, A.W., Gore, J.C., Buchanan, R.J., Constable, R.T., Hawkins, K.A., Sahay, N. and Krystal, J.H. (2007) Probing the pathophysiology of auditory/verbal hallucinations by combining functional magnetic resonance imaging and transcranial magnetic stimulation. Cerebral Cortex, 11, 27332743. http://dx.doi.org/10.1093/cercor/bhl183

[14] Fitzgerald, P.B., Benitez, J., Daskalakis, J.Z., Brown, T.L., Marston, N.A.U., de Castella, A., et al. (2005) A double-blind sham-controlled trial of repetitive transcranial magnetic stimulation in the treatment of refractory auditory hallucinations. Journal of Clinical Psychopharmacology, 25, 358-362. http://dx.doi.org/10.1097/01.jcp.0000168487.22140.7f

[15] Brunelin, J., Poulet, E., Bediou, B., Kallel, L., Dalery, J, D'Amato, T., et al. (2006) Low frequency repetitive transcranial magnetic stimulation improvessource monitoring deficit in hallucinating patients with schizophrenia. Schizophrenia Research, 81, 41-45. http://dx.doi.org/10.1016/j.schres.2005.10.009

[16] McIntosh, A.M., Semple, D., Tasker, K., Harrison, L.K., Owens, D.G.C., Johnstone, E.C., et al. (2004) Transcranial magnetic stimulation for auditory hallucinations in schizophrenia. Psychiatry Research, 127, 9-17. http://dx.doi.org/10.1016/j.psychres.2004.03.005

[17] De Jesus, D.R., Gil, A., Barbosa, L., Lobato, M.I., Magalhães, P.V., Favalli, G.P., Marcolin, M.A., Daskalakis, Z.J. and Belmonte-de-Abreu Pda, S. (2011) A pilot doubleblind sham-controlled trial of repetitive transcranial magnetic stimulation for patients with refractory schizophrenia treated with clozapine. Psychiatry Research, 188, 203-207. http://dx.doi.org/10.1016/j.psychres.2010.11.022

[18] Huang, Y.Z., Edwards, M.J., Rounis, E., Bhatia, K.P. and Rothwell, J.C. (2005) Theta burst stimulation of the human motor cortex. Neuron, 45, 201-206. http://dx.doi.org/10.1016/j.neuron.2004.12.033

[19] Sommer, I.E.C., Diederen, K.M.J., Blom, J.-D., Willems, A., Kushan, L., Slotema, K., Boks, M.P., Daalman, K., Hoek, H.W., Neggers, S.F. and Kahn, R.S. (2008) Auditory verbal hallucinations predominantly activate the right inferior frontal area. Brain, 131, 3169-3168. http://dx.doi.org/10.1093/brain/awn251 